
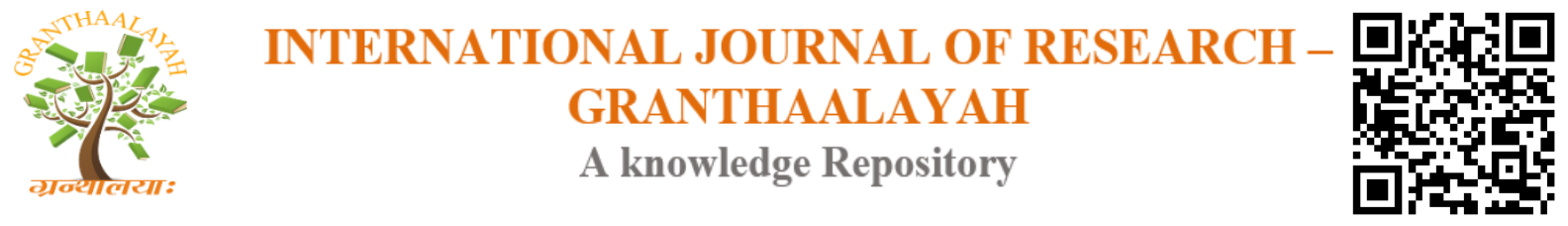

Science

\title{
FAVIPIRAVIR AS A POTENTIAL DRUG IN THE TREATMENT OF COVID-19
}

\author{
Dany Geraldo Kramer *1, Maria Josilene Leonardo Da Silva ${ }^{2}$, Gislanne Stéphanne \\ Estevam Da Silva ${ }^{2}$, Ana Maria Marinho Andrade De Moura ${ }^{3}$, Geraldo Barroso \\ Cavalcanti Junior ${ }^{4}$, Anesio Mendes De Sousa ${ }^{5}$, Ana Elizabeth Alves Da Silva ${ }^{6}$ \\ ${ }^{1}$ Professor Dr. Postgraduate Program in Family Health (RENASF) - Federal University of Rio \\ Grande do Norte \\ ${ }^{2}$ Student. Federal University of Rio Grande do Norte. Brazil \\ ${ }^{3}$ Professor MsC. Federal University of Rio Grande do Norte. Brazil \\ ${ }^{4}$ Professor Dr. Federal University of Rio Grande do Norte. Brazil \\ ${ }^{5}$ Professor MSc. Federal Institute of Education, Science and Technology of Tocantins \\ ${ }^{6}$ Professor Dra Estácio University. Brazil
}

\begin{abstract}
Favipiravir is a drug developed for use against influenza and has been used successfully in other infectious conditions. After being internalized in the cell, the substance is phosphoribosylated acting on the RNA polymerase, and thus inhibiting replication and RNA viruses. Thus, the present study aimed to discuss the potential use of favipiravir in coronovavirus infections. There have been few studies involving favipiravir in COVID 19, however there is a report of recovery in more than $70 \%$ of patients diagnosed with pneumonia. However, new studies need to be carried out, mainly randomized clinical trials, so that the potential use of favipiravir in coronaviruses is adequately grounded.
\end{abstract}

Keywords: Favipiravir; Coronavirus; Therapy.

Cite This Article: Dany Geraldo Kramer, Maria Josilene Leonardo Da Silva, Gislanne Stéphanne Estevam Da Silva; Ana Maria Marinho Andrade De Moura, Geraldo Barroso Cavalcanti Junior, Anesio Mendes De Sousa, and Ana Elizabeth Alves Da Silva. (2020). "FAVIPIRAVIR AS A POTENTIAL DRUG IN THE TREATMENT OF COVID-19." International Journal of Research - Granthaalayah, 8(4), 7-12. https://doi.org/10.29121/granthaalayah.v8.i4.2020.2.

\section{Introduction}

Favipiravir is an antiviral developed in Japan in 2014, as alternatives for the treatment of flu infections, mainly influenza. In recent years, this drug has been used in several infectious outbreaks around the world, including infections by the Ebola, Lassa, Marburg and Nipah virus $[1,2,3]$. 
Favipiravir acts against several RNA viruses, as it is converted intracellularly into a phosphoribosylated structure, which is captured by viral RNA polymerase, thereby blocking viral replication. This drug is already approved by the Japanese government, and the potential application in the COVID-19 pandemic is relevant, as it has already gone through all the drug development procedures, ensuring clinical safety studies and availability produced in the market $[1,4,5,6]$.

The COVID-19 is a coronavirus that has caused hundreds of thousands of infections and tens of thousands of deaths around the world, being considered an RNA virus that is transmitted by respiratory droplets or by food and objects contaminated by viral particles $[7,8,9,10]$.

However, due to the clinical severity and lack of therapeutic option, the use of favipiravir has been studied for its potential use in the treatment of COVID -19. This drug presents as a dosage regimen in the treatment for Influenza with application on the first day of $1600 \mathrm{mg}$, twice daily, and sequence of $600 \mathrm{mg}$, twice daily, between 03 to 07 days [1, 5]. In this sense, the present study aimed to discuss the potential use of favipiravir in coronavirus infections.

\section{Coronavirus - Covid 19}

Coronaviruses are responsible for infections in mammals, birds and humans, causing respiratory, enteric and renal clinical conditions. In the last decades they have been responsible for infectious conditions in thousands of people, the most recent being Severe Acute Respiratory Syndrome (SARS-CoV) in 2003, Middle East Respiratory Syndrome (MERS-CoV) in 2012 and the New Coronavirus pandemic (COVID 19 ) that started in China [10, 11, 12].

These are viruses (Figure 01) enveloped with a single-stranded RNA genome, and may be pleomorphic or spherical. The structure of the viral envelope is formed by a lipid bilayer, where hemagglutinin and glycoprotein $\mathrm{S}$ are anchored, essential structures for the virus to bind to the host cell $[7,13,14]$.

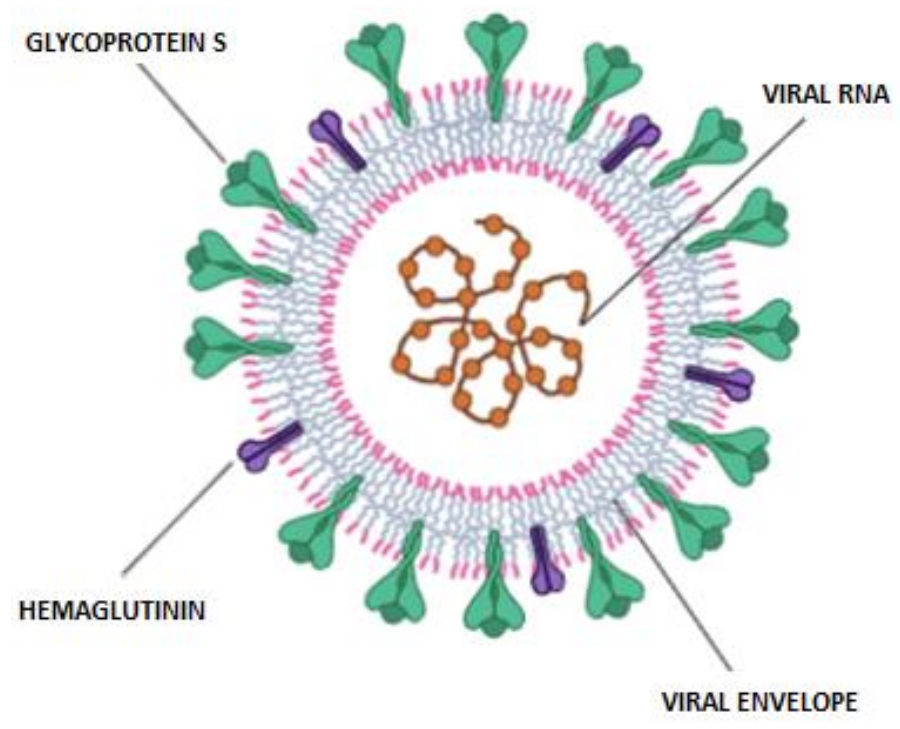

Figure 1: Basic structure of the coronavirus. "Created with BioRender.com" 
Transmission from human to human often occurs in close contact, mainly through respiratory droplets expelled by infected (sneeze / cough). These droplets can also be deposited on the hands, objects and food for later transmission $[7,14,15]$.

The COVID -19 then interacts with the host cell receptor through glycoprotein (S), inducing viral internalization through fusion with the host cell surface or formation of endocytic vesicles (Figure 02). This merges with the lysosome that will act on the viral structure and subsequent release of genomic RNA into the cytoplasm. The RNA will be translated, producing a polyprotein that will give rise to an RNA polymerase. Through the action of RNA polymerase, subgenomic mRNAs will be generated, being translated into structural viral proteins. In the final step, the $\mathrm{S}$ glycoprotein is expressed on the cell membrane surface and the virus is assembled inside vesicles. Vesicles are enveloped by the envelope containing glycoprotein $S$ and release viruses by exocytosis $[7,9,10$, 13].

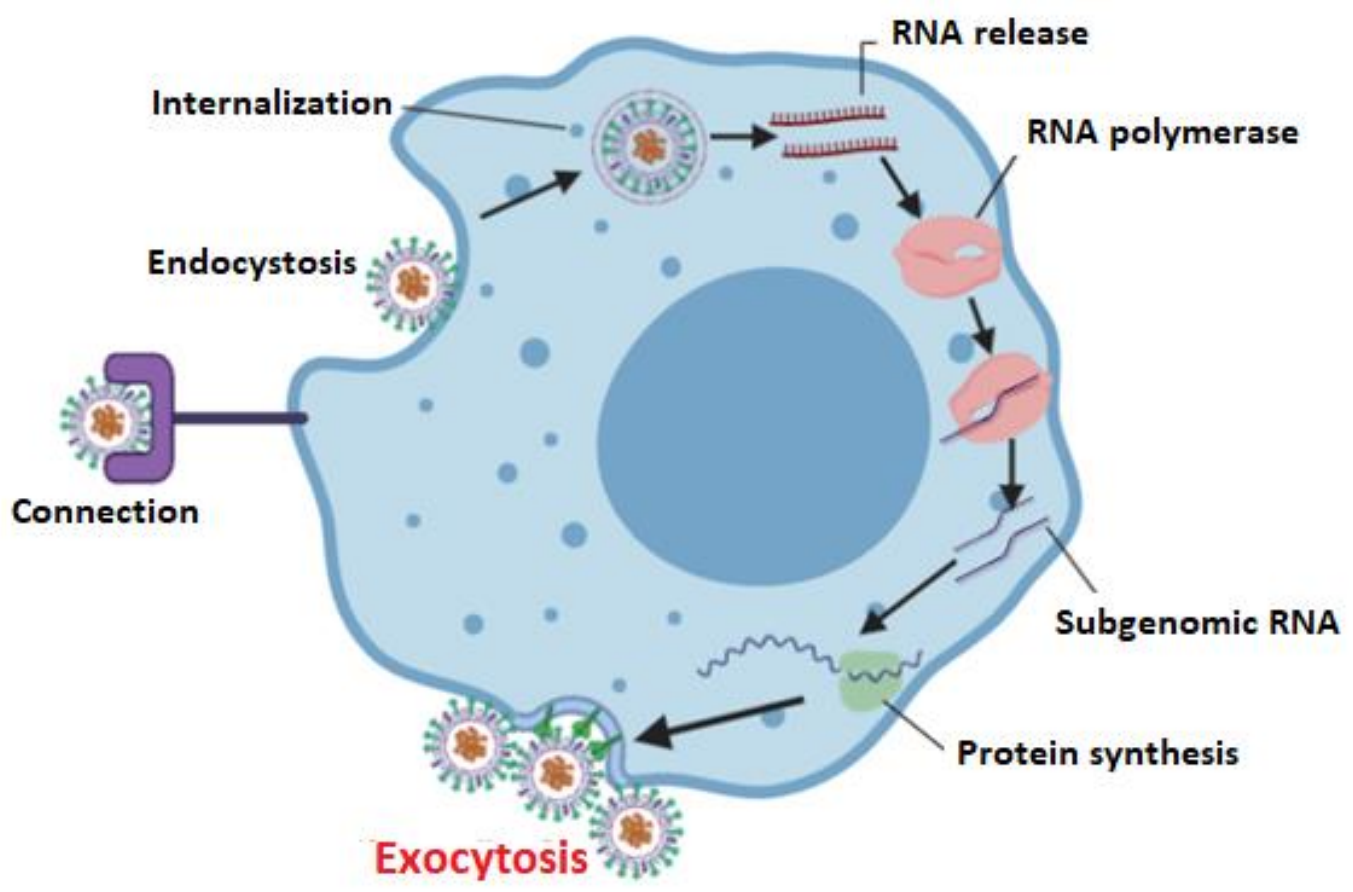

Figure 2: Schematic representation of coronavirus replication "Created with BioRender.com"

The incubation period varies between 02 to 14 days for the appearance of the first symptoms, the most common being fever, cough and dyspnoea. When the patient has comorbidities, such as underlying diabetes mellitus, hypertension and cardiovascular diseases, the clinical evolution can be more severe, resulting in severe dyspnea, viral pneumonia, acute cardiac injury, secondary infections, pneumothorax and renal impairment. Often leading the patient to ICU admission, they may need renal and ventilatory support $[7,8,10,11]$.

In a study by Wang et al. [15], 138 patients diagnosed with pneumonia by COVID 19 were followed, with a mean age of 56 years (42-68). They had the most common symptoms: fever (98.6\%), fatigue (69.6\%) and dry cough (59.4\%). Of these, 26\% of patients required admission to the intensive care unit and $4.3 \%$ died. Most patients received antibacterial therapy and 
glucocorticoid therapy as a result of complications. Chen et al. [16] followed 99 patients who had fever (83\%), cough (82\%), dyspnea (31\%), myalgia (11\%) and mental confusion (09\%), as the most common symptoms. Of these, $11 \%$ developed complications and died. The lack of specific therapy hinders the reversal of more severe conditions, demonstrating the importance of analyzing favipiravir with a punctual drug in the treatment of COVID 19.

\section{Favipiravir X Covid 19}

Favipiravir $\left(\mathrm{C}_{5} \mathrm{H}_{4} \mathrm{FN}_{3} \mathrm{O}_{2}\right)$ is a derivative of pyrazinecarboxamide that was created in 2014 by the company Toyama Chemical (Japan) as a new therapeutic option for respiratory infections against RNA viruses. It presents rapid absorption after oral administration, ensuring high bioavailability above $90 \%$. Most of this drug is excreted via the kidney (DELANG et al., 2018; JANOWSKI et al., 2020; SHIKARI et al., 2020; DRUGBANK, 2020).

This medicine has been used in the treatment of viral infections (RNA), such as Ebola, Lassa, Marburg and Nipah. It presents itself as a drug that is generally well tolerated and may result in mild gastrointestinal discomfort. In cases of overdose, nausea, vomiting and decreased locomotor activity may be observed. In favipiravir development studies, teratogenic properties were found, and should be avoided in pregnant women $[1,2,3,17,18]$.

The potential application in the treatment of COVID-19 is explained because this drug is converted intracellularly into its active phosphoribosylated form (ribofuranosyltriphosphate - RFTS) - Figure 03, this substance is recognized as a substrate by viral RNA polymerase. Following the RNA polymerase activity is compromised, blocking the synthesis of subgenomic mRNAs, and the translation of structural viral proteins, resulting in inhibited viral replication $[1,3,4,5,17,19]$.

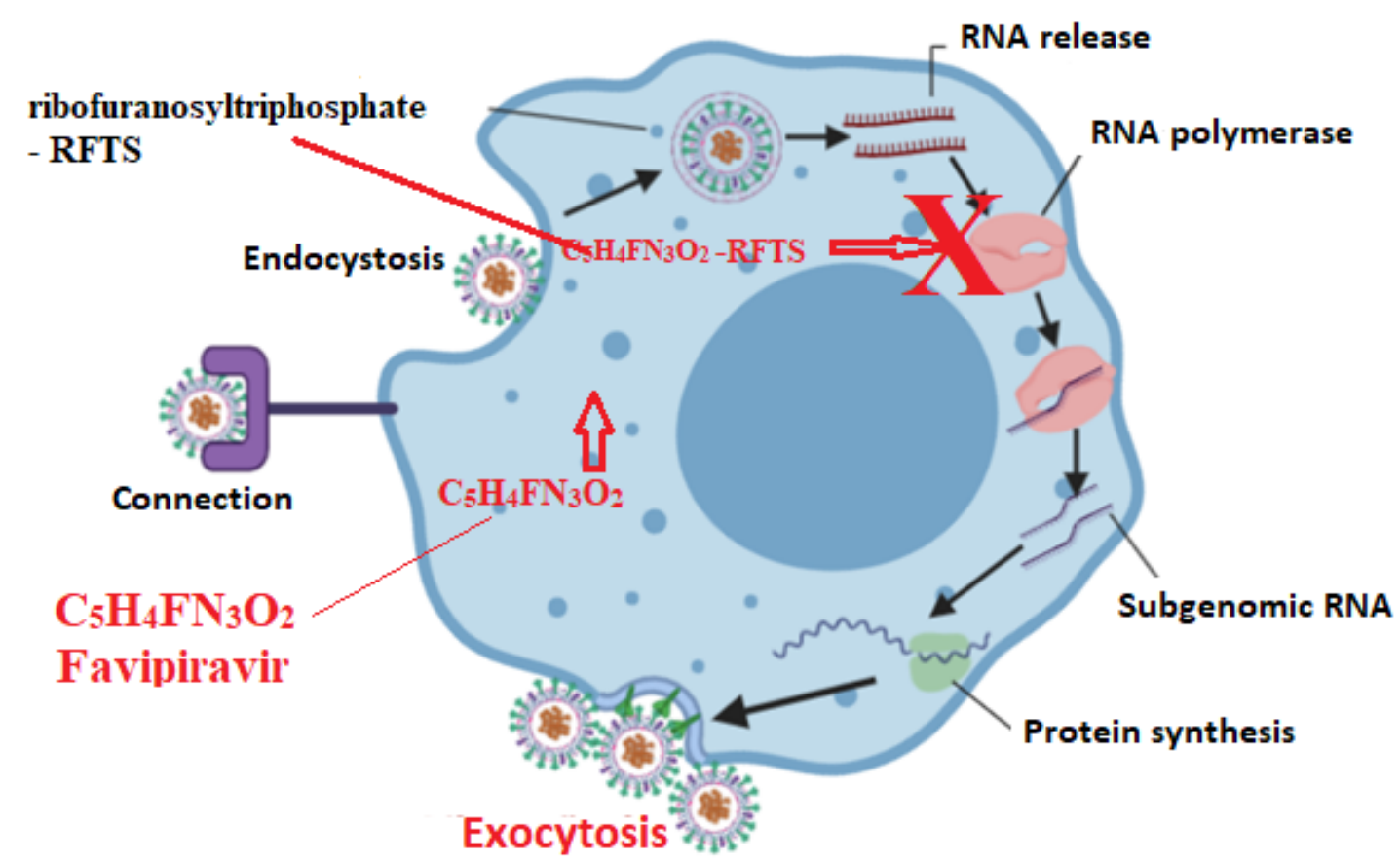

Figure 3: Mechanism of action of favipiravir. "Created with BioRender.com" 
Studies involving the application of favipiravir in coronoviruses are still few, one of which was developed by Chen et al. [16] who followed, 120 patients diagnosed with pneumonia by COVID -19. These were treated, following the conventional protocol of this drug (1,600 mg, twice a day, on the day), following maintenance doses, twice a day, 600mg, twice, with use between 07 and 10 days. The recovery of $71.43 \%$ of the patients treated with favipiravir was observed, being considered a potential drug for application in the therapy of COVID-19.

Studies on the use of this drug in viral infections (RNA) are well established, however its use for the treatment of COVID-19, is still little known. Although, as previously described, use contributed to the recovery of $71.43 \%$ of patients with pneumonia from COVID 19, new randomized, blinded clinical trials should be described, in order to better understand the effectiveness of favipiravir in the current pandemic. This would be important for countries like Brazil, to evaluate the liberation of the manufacture and commercialization of this drug in their territories. Still, some contraindications must be observed, such as use in pregnant women, since this drug is teratogenic $[1,7,9]$.

A study between the Clinical Medical Research Center of the National Infectious Diseases and the Third People's Hospital of Shenzhen involving 80 patients (including the experimental group and the control group) should that favipiravir had better outcomes in comparison to lopinavir and ritonavir. Also, the medication presented fewer adverse reactions in a significant way, than of lopiravir and ritonavir (20).

Therefore, although favipiravir is well tolerated by patients, use in some countries has already been approved, studies should be further developed to better support its application in coronaviruses.

\section{Conclusions}

It is concluded from the data collected, that there are promising results in the use of Favipiravir in COVID-19. However, new studies need to be carried out, mainly randomized clinical trials, so that the potential use of favipiravir in coronaviruses is adequately grounded. Thus, based on new studies, countries like Brazil will be able to evaluate its effectiveness and release for commercialization in their territories and guide future clinical interventions.

\section{Recommendations}

Therefore, although favipiravir is well tolerated by patients, use in some countries has already been approved, studies should be further developed to better support its application in coronaviruses.

\section{References}

[1] Chang, C.; jianying, H.; zhenshun, C. M. Favipiravir versus Arbidol for COVID-19: A Randomized Clinical Trial. PubPeer, 31 Mar 2020.

[2] Furuta, Y.; komeno, T.; komeno, T. Nakaruma, N. Favipiravir (T-705), a broad spectrum inhibitor of viral RNA polymerase. Proc Jpn Acad Ser B Phys Biol Sci. Aug 2; 93(7): 449-463. 2017.

[3] Madelain, V.; mentre, F.; baize, S. Modeling favipiravir antiviral efficacy against emerging viruses: from animal studies to clinical trials. Pharmacometrics \& Systems Pharmacology, 20 March 2020 
[4] Bricker, T. L.; shafiuddin, M.; gounder, A. P. Therapeutic efficacy of favipiravir against Bourbon virus in mice. PLoS Pathog. 2019 Jun; 15(6).

[5] Drugbank. Favipiravir. Disponível em: https://www.drugbank.ca/drugs/DB12466. Acessado em 02/04/2020.

[6] Goldhilla, D. H.; velthuisc, A. J. W. Fletcher, R. A. The mechanism of resistance to favipiravir in influenza. PNAS | November 6| vol. 115, 2018.

[7] AdhikarI, S. P.; meng, S.; wu, Y.; mao, Y. Epidemiology, causes, clinical manifestation and diagnosis, prevention and control of coronavirus disease (COVID-19) during the early outbreak period: a scoping review. Infectious Diseases Of Poverty, [s.1.], v. 9, n. 1, p.9-29, 17 mar. 2020.

[8] Who, World Health Organization. Coronavirus disease 2019 (COVID-19) Situation Report...3.14. 2020.

[9] Li, G.; clerc, E. D. Therapeutic options for the 2019 novel coronavirus (2019-nCoV), Nature Review, 10, 2020

[10] Sahin, A.R.; erdogan, A.; dineri, Y. Novel Coronavirus (COVID-19) Outbreak: A Review of the Current Literature. EJMO;4(1):1-7. 2020.

[11] Rui, W.; liao, C.; he, H.; hu, C. COVID-19 in Hemodialysis Patients: A Report of 5 Cases. American Journal of Kidney Diseases Available online 31 March 2020.

[12] Li, Q.; guan, X.; wu, P.. Early Transmission Dynamics in Wuhan, China, of Novel CoronavirusInfected Pneumonia. N Engl J Med; published online Jan 29, 2020.

[13] Guo, Y. R.; cao, Q. D.; hong, Z. S. The origin, transmission and clinical therapies on coronavirus disease 2019 (COVID-19) outbreak - an update on the status. Military Medical Research, [s.l.], v. 7, n. 1, p.2-10, 13 March. 2020.

[14] Praja, M.; sarma, P.; shekhar, N.. Drug targets for corona virus: A systematic review. Indian Journal of Pharmacology (Vol. 52, Issue 1), 2020.

[15] Wang, D.; hu, B.; hu. C. Clinical Characteristics of 138 Hospitalized Patients With 2019 Novel Coronavirus-Infected Pneumonia in Wuhan, China. JAMA. 2020;323(11):1061-1069

[16] Dawei W.; bo, H.; chang, H. Clinical Characteristics of 138 Hospitalized Patients With 2019 Novel Coronavirus-Infected Pneumonia in Wuhan, China. JAMA. ;323(11):1061-1069. 2020

[17] Chen, N.; Zhou, M.; Dong, X.; Qu, J.; Gong, F.; Han, Y.; Qiu, Y.; Wang, J.; Liu, Y.; Wei, Y.; et al. Epidemiological and clinical characteristics of 99 cases of 2019 novel coronavirus pneumonia in Wuhan, China: A descriptive study. Lancet 2020, 6736, 1-7

[18] Delang, L.; abdelnabi, R.; nets, J. Favipiravir as a potential countermeasure against neglected and emerging RNA viroses. Antiviral Research Volume 153, May Pages 85-94, 2018.

[19] Janowski, A. B.; dudley, H..; wang, D. Antiviral activity of ribavirin and favipiravir against human astroviruses. Journal of Clinical Virology Volume 123, February, 2020.

[20] Shikari, K.; daikoku, T.; cheng, C. Favipiravir, an anti-influenza drug against life-threatening RNA virus infections. Pharmacology \& Therapeutics 22 February 2020.

[21] Dong, L.; Hu, S.; Gao, J. Discovering drugs to treat coronavirus disease 2019 (COVID-19). Drug Discoveries \&Therapeutics.; 14(1)58-60) 2020.

*Corresponding author.

E-mail address: dgkcs@ yahoo.com.br 